\title{
Material flow during the extrusion of simple and complex cross-sections using FEM
}

\section{Flitta*1 and T. Sheppard ${ }^{1}$}

This paper deals with the extrusion of rod and shape sections and uses a 3D finite element model analysis (FEM) to predict the effect of die geometry on maximum extrusion load. A description of material flow in the container is considered in more detail for rod and shape sections in order to fully comprehend the transient conditions occurring during the process cycle. A comparison with experiments is made to assess the relative importance of some extrusion parameters in the extrusion process and to ensure that the numerical discretisation yields a realistic simulation of the process. The usefulness and the limitation of FEM are discussed when modelling complex shapes. Results are presented for velocity contours and shear stress distribution during the extrusion process. It is shown that for most of the shapes investigated, the material making up the extrudate cross-sections originates from differing regions of virgin material within the billet. The outside surface of the extrudate originates from the material moving along the dead metal zone (DMZ) and the core of the extrudate from the central deformation zone. The FE program appears to predict all the major characteristics of the flow observed macroscopically.

Keywords: Extrusion, Aluminium, FEM, Material flow, Shape sections

\section{Introduction}

Complex sections that have non-symmetric shapes are produced by the extrusion process. In this communication, several simple shapes including $\mathrm{T}, \mathrm{U}$ and square sections are investigated.

It is generally known that a large increase in the extrusion load is required to extrude a section of these types of complexities containing re-entrant corners or thin fin sections, over that of simple circular crosssections. Sheppard ${ }^{1}$ and Sheppard and Wood ${ }^{2}$ reported in more detail the parameter that can represent the degree of difficulty of extrusion of complex shapes by the use of a shape factor or peripheral ratio.

The material flow is also of particular importance when extruding complicated sections. The occurrences of material flow during the extrusion process have been the focus of much interest in aluminium alloys in general $^{3-8}$ but has been most often limited to rod extrusions. The influence of section geometry on the deformation zone is difficult to establish, unlike axisymmetric rod extrusion where the circumferential strain is essentially zero, the introduction of a third dimensional strain requires more careful interpretation. This is especially so near the die throat where the extrudate surface and structural features are generated. Studies of the material flow during extrusion are well documented in literature. They range from the most

Bournemouth University, Studland House, 12 Christchurch Road, Bournemouth, BH1 3NA, Dorset, UK

*Corresponding author, email iflitta@ bournemouth.ac.uk commonly used technique such as the gridded billet, ${ }^{6-8}$ introducing pins of an aluminium alloy into the as-cast billet and then grinding and etching the surface after the end of the extrusion, ${ }^{5,9,10}$ to marking grids within the initial billet. ${ }^{3,4,11}$ However, the gridded billet technique relies on planar flow, and is not suitable to study material flow for complex cross-sections. The pins or volume element technique is more successful than the gridded billet technique. The advantage of this technique is that the information cannot be erased in regions of heavy shear. However, the process itself is difficult to analyse owing to many re-entrant corners and the rotational component of velocity that can cause unpredictable behaviour of the metal flow during extrusion and may require more than one section plane to represent the true material flow. Therefore, numerical simulations are necessary to gain more insight during the extrusion process cycle because of the ease with which FEM is able to predict the material flow from the beginning to the end of the extrusion. However, a false assumption of the extrusion being a steady state deformation is usually applied and Eulerian formulation based FEM codes are used. In order to investigate the microstructural evolution and predict the extrudate properties, the material history must be preserved during the process cycle. Therefore, a Lagrangian formulation based FEM must be adopted.

\section{FEM programs and formulations}

In the present work all the simulations were conducted using the French developed software Forge2 and Forge 3 from Transvalor SA (St Michel, Paris, France). 
Originally this package was developed at Ecole des Mines de Paris and in the past decade has successfully been applied to 2D and 3D FEM simulation on various forming processes., ${ }^{3,41-15}$ In addition to the plastic deformation of the work piece, Forge 2 software is capable of coupling the temperature calculation of both the work piece and the die(s), and of incorporating the elastic deformation of the die(s). The automatic remeshing technique enables the simulation of very complex parts. The ability of the solver to use multi-processors significantly cuts the analysis time.

The model used for this simulation is described by Chenot. ${ }^{16}$ The data structure of the program include, the governing equations, the finite element of the work piece, the rheology of the material, the tooling description, the frictional interface, and the numerical parameters to be included in the calculation code. The program uses implicit FEM to calculate the hot working parameters: load, strain rate, temperature rise and deformation history.

In modelling of the extrusion process, the governing equations are strongly non-linear. These non-linearities are due to complex constitutive equations, non-linear kinematics and contact. The vast majority of approaches for solving systems of non-linear equations are based on the Newton-Raphson iterative scheme or its variants.

In the simulation of the extrusion process, material history and contact phenomena play an important role. Therefore, the best suited approach for the numerical simulation of extrusion process is the Lagrangian method. The advantage of tracking the history of the material point or a node, for surface formation, microstructure evolution and temperature evolution are of paramount importance to the process optimisation. Therefore a Lagrangian method is adopted for the program which can therefore accurately define the material properties, state variables and boundary conditions.

\section{Governing equation}

The FEM used in metal forming can be generally categorised into viscoplastic and elastic viscoplastic FEM, depending on which material constitutive equations are used. Constitutive equations for plastic deformations are usually based on rate equations. For large deformation processes, i.e. extrusion, the elastic deformations are totally ignored (the elastic part of the strain rate can be neglected). That means that a calculated strain increment equals the plastic strain increment.

For the extrusion of hot aluminium alloys, viscoplastic FEM is adopted to simulate the process because plastic strain dominates the process. The most economical laws are purely viscoplastic approximations. The most widely used is that proposed by Sellars and Tegart ${ }^{17}$ and subsequently modified by Sheppard and Wright $^{18}$ to yield the steady state flow stress $\bar{\sigma}$ from the equation

$$
Z=A[\sinh (\alpha \dot{\sigma})]^{\mathrm{n}}=\stackrel{:}{\varepsilon} \exp \left(\frac{\Delta H}{G T}\right)
$$

from which

$$
\dot{\sigma}=\frac{1}{\alpha} \ln \left\{\left(\frac{Z}{A}\right)^{1 / \mathrm{n}}+\sqrt{\left(\frac{Z}{A}\right)^{2 / \mathrm{n}}+1}\right\}
$$

in which $Z\left(\mathrm{~s}^{-1}\right)$ is termed the temperature compensated strain rate. The Arhenius term indicates that hot working is a thermally activated process ${ }^{19}$ with $\Delta H$ $\left(\mathrm{kJ} \mathrm{mol}^{-1}\right)$ as the activation energy for deformation, $\dot{\bar{\varepsilon}}$ $\left(\mathrm{s}^{-1}\right)$ is the mean equivalent strain rate and $G$ $\left(8 \cdot 314 \mathrm{~J} \mathrm{~mol}^{-1} \mathrm{~K}^{-1}\right)$ is the universal gas constant. $A$ $\left(\mathrm{s}^{-1}\right), \alpha\left(\mathrm{m}^{2} \mathrm{MN}^{-1}\right)$ and $\mathrm{n}$ are constants within the extrudable temperature range; $n$ is generally assumed to be a measure of the strain rate sensitivity and $\alpha$ appears to be constant describing the transition from power to exponential relationships for the flow stress. The remaining terms in the equation require definition because the strain rate is specific to extrusion geometry and the temperature $T(\mathrm{~K})$ varies throughout the extrusion ram stroke. The activation $(\Delta H)$ energy is considered as a material constant in the hot deformation range and is usually close to the value for self diffusion of the alloy.

The constitutive equation is incorporated into the solution by programming the user subroutine.

The temperature evolution in the deformation zone is governed by internal heat conduction and internal heat dissipation under the constraints defined on the area boundary in terms of interchange (radiation, conduction and convection) or in terms of imposed temperature. This evolution is represented by the following classical heat equation

$$
\rho c \frac{\mathrm{d} T}{\mathrm{~d} t}=\operatorname{div}(k \operatorname{grad}(T))+\dot{q}_{\mathrm{v}}
$$

where $\rho$ is the density, $c$ the specific heat, $k$ is the conductivity, $T$ is the temperature and $\dot{q}_{\mathrm{v}}$ is the internal heat dissipation generated by the plastic deformation. The terms $\rho c \frac{\mathrm{d} T}{\mathrm{~d} t}$ and $\operatorname{div}(k \operatorname{grad}(T))$, are the temperature evolution and, internal conduction respectively.

On the free surface, radiation is modelled by

$$
-k \frac{\partial T}{\partial n}=\varepsilon_{\mathrm{r}} \sigma_{\mathrm{r}}\left(T^{4}-T_{0}^{4}\right)
$$

where $\varepsilon_{\mathrm{r}}$ is the material emissivity in its current condition, $\sigma_{\mathrm{r}}$ the Stephan's constant, $T$ is the area boundary local temperature and $T_{0}$ is the outside temperature.

In addition to the above governing equations, boundary conditions to describe geometry and friction are required. For an accurate simulation of the extrusion process, the friction coefficient must be identified continuously during the process cycle. This is not only required for press capacity, but also to define the temperature changes occurring in the material. In the simulation of the extrusion process, the friction coefficient, as shown by Flitta and Sheppard ${ }^{4,11}$ has a great effect on the computed load and the temperature evolution. In addition to that, it has also great effect on the computed results, such as material flow, microstructural evolution and the product surface quality. Friction conditions at the billet/tool interface are known to affect the flow paths of the material at the container and through the die by changing the extrusion parameters, loads, stresses and surface quality. Using different friction conditions and various input temperatures permits the extrusion parameters to be predicted from the simulation. These changes are the consequences of an increase in the overall billet temperature 


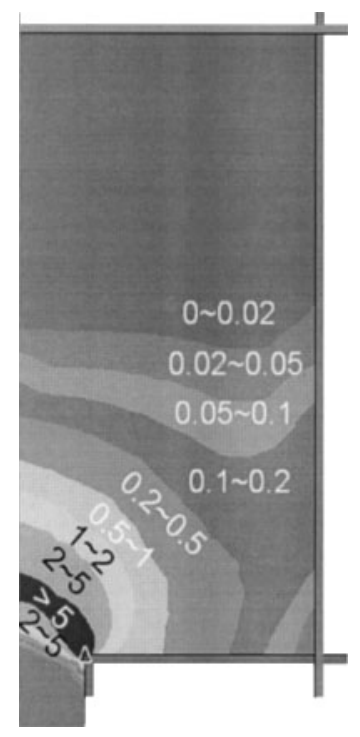

1 Boundary area where the values of $Z_{d}$ are calculated

within the deformation zone and the decrease of the area of contact with further ram travel. ${ }^{4,11}$

Friction is dealt with by assuming a modified Tresca criteria law ${ }^{4}$ and is written as follows

$$
\tau=-\bar{m}_{\Delta \mathrm{L}_{\mathrm{n}}} \frac{\sigma_{0}}{\sqrt{3}}
$$

where

$$
m_{\Delta \mathrm{L}_{\mathrm{n}}}=\left[A+\alpha n \ln \left(Z_{\mathrm{d}} / A\right)+B\right]_{\Delta \mathrm{L}_{\mathrm{n}-1}}
$$

and $\tau$ is the shear stress at the interface, $\sigma_{0}$ represents the yield stress and $\sigma_{0} / \sqrt{3}$ is usually termed the shear flow stress. $\bar{m}_{\Delta \mathrm{L}_{\mathrm{n}}}$ is the friction factor and varies with the ram stroke where $\Delta L_{\mathrm{n}}$, is incremental ram travel (i.e. each $2.5 \mathrm{~mm}$ of ram displacement). $Z_{\mathrm{d}}$, the average of the Zener-Hollomon parameter defined in equation (1), contains the data describing the alloy behaviour, ram speed and temperature. During the process, the maximum deformation is expected to occur at the re-entrant corner near the die orifice as illustrated in Fig. 1. Therefore the average strain rate of the whole deformation area could not reflect the true condition of the process, so the average strain rate of the area with $\ln (\dot{\varepsilon})>0.5$ was used to calculate the values of $Z_{\mathrm{d}}$. The remaining terms $A, n$ and $\alpha$ are defined in equation (1).

The variation of friction conditions during the extrusion cycle relating to the process conditions is written in the software algorithm as follows

$$
\dot{m}=-0 \cdot 6011 \alpha n \ln \left(Z_{\mathrm{d}} / A\right)+1 \cdot 1261
$$

just before the peak pressure is established and

$$
m=-0 \cdot 4973 \alpha n \ln \left(Z_{\mathrm{d}} / A\right)+1 \cdot 1056
$$

after the dead metal zone (DMZ) is fully established.

\section{Simulation considerations}

The definition of the tooling is one of the essential data inputs that have to be integrated into the simulation calculation. Consequently, both for the geometry (the

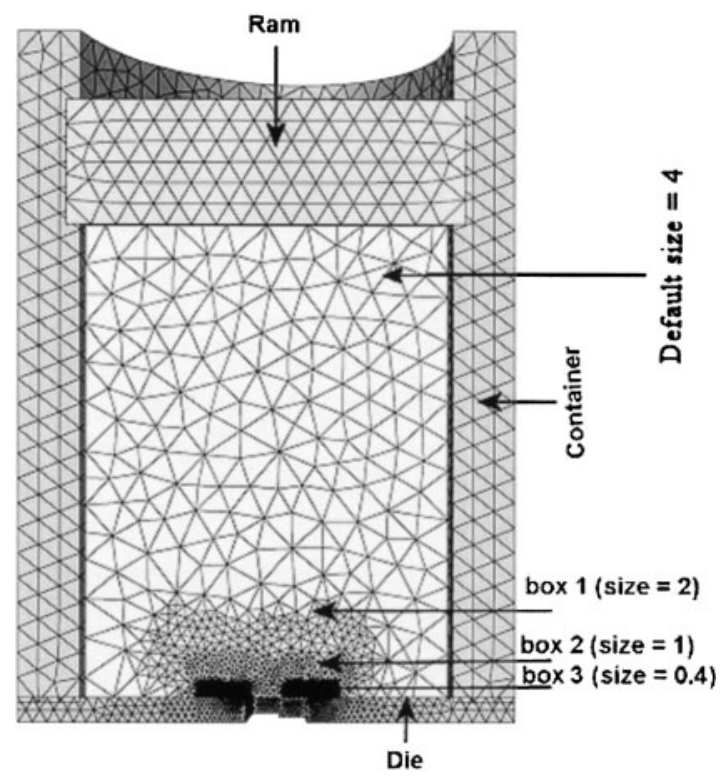

2 Meshing of the billet tool and setup configuration prior to simulation in Forge3

precision of the boundary) and the kinematics (to follow the mechanical deformation), simulation must be designed accurately.

During the simulation of extrusion, large deformations are predominant. Therefore, complete remeshing which is mandatory in areas of excessive deformation is a source of loss/gain of volume. The elements become severely distorted during the process and consequently, the need for remeshing is necessary to continue the simulation. The frequency of remeshing is controlled by the degree of deformation and is a user variable.

In Forge3, the meshing is based on two concepts: the quality of the elements and the shape preservation (geometry). These two concepts are quantified and controlled by the user. The strategy to obtain the 'best' mesh is based on node addition, suppression and regularisation to improve an existing given surface or to rebuild a new surface from an initial one. These should be done without modifying the domain geometry (shape preservation). In order to generate a volume mesh, the program requires an initial triangular surface mesh with three node linear triangles. The quality of this volume mesh depends on the quality of the billet surface mesh generated in a CAD system (I-DEAS, Pro-Engineer, etc.).

\section{Pre-processing}

\section{FEM models and source of experimental data}

The tooling geometry of the shape dies and the billets were modelled using the I-DEAS Master-Series software. Taking advantage of the symmetry planes in the design and in order to reduce computation time, half of the models were constructed for $\mathrm{T}$ and $\mathrm{U}$ shapes. The square shape was constructed using only a quarter of symmetry of the models. A surface mesh (2D) was then generated using three node linear triangles corresponding to the outer surface of the tooling and the billet. Once these models were built and meshed in 2D, the finite element model information was imported by Forge 3 program algorithm in order to check the surface quality of the tools and to generate the $3 \mathrm{D}$ volume mesh 


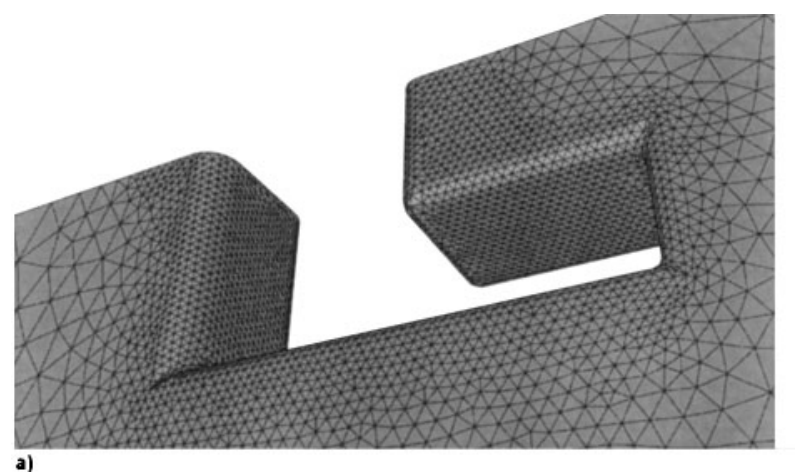

a)

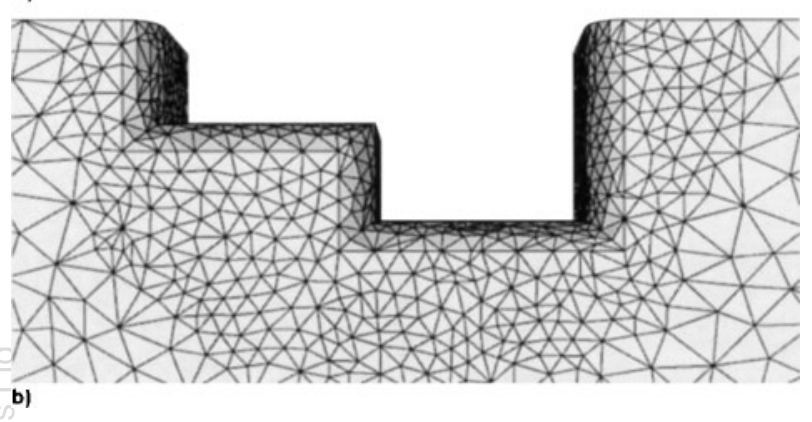

$a$ 'U' section; $b$ ' $T$ ' section

3 CAD model and mesh of the outer surfaces of section shapes

for the billet using three node tetrahedral elements as shown in Fig. 2. To control the degree of remeshing in the areas where high deformation is expected, fine refinement mesh boxes of an Eulerian type (but maintaining Lagrangian flow) were applied to the billet. The remeshing values are controlled by an average aimed size of an edge of an element and the distribution is specified by 'mesh boxes', i.e. boxes that define regions of the mesh on which a mesh size is imposed during the computation. Refinement mesh boxes for the $\mathrm{T}, \mathrm{U}$ and square shapes were defined and applied at the re-entrant corners where high deformation is expected as illustrated in Fig. 3. The average initial mesh size in the billet is $4 \mathrm{~mm}$.

The chemical compositions of the alloy used in this investigation are given in Table 1. All the experimental data are extracted from the literature. ${ }^{2,10,20}$ Experiments were performed on a $5 \mathrm{MN}$ vertically mounted extrusion press with a heated container.

The rheological data input for the shapes investigated are summarised in Tables 2 and 3. The dimensions of the billet are $75 \mathrm{~mm}$ diameter and $95 \mathrm{~mm}$ length. The initial container temperature was $50^{\circ} \mathrm{C}$ below that of the initial billet temperature to simulate industrial conditions. ${ }^{1}$ The billet was extruded at a constant ram speed of $3 \mathrm{~mm} \mathrm{~s}^{-1}$.

The data describing the material behaviour available to the authors were obtained using torque-twist data from torsion tests and optimising to obtain the form of equation (1). Simulations were conducted on a Dell dual processor workstation.

Table 1 Chemical composition of aluminium alloys, wt- ${ }^{1}$

\begin{tabular}{lllllllll}
\hline Alloy & Al & $\mathrm{Cu}$ & $\mathrm{Mg}$ & $\mathrm{Fe}$ & $\mathrm{Si}$ & $\mathrm{Mn}$ & $\mathrm{Ti}$ & $\mathrm{Zn}$ \\
\hline AA 2024 & Bal & $3.8-4.9$ & $1.2-1.8$ & 0.5 & 0.5 & $0.3-0.9$ & 0.15 & 0.25
\end{tabular}
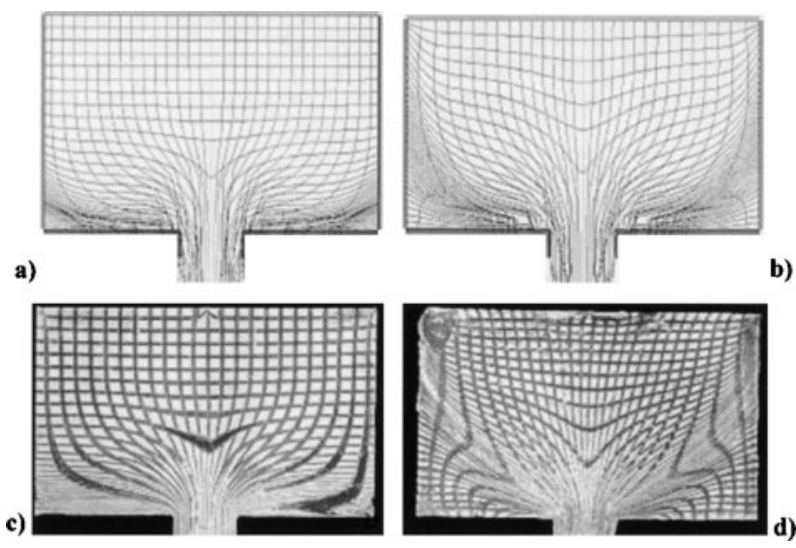

a flow at $300^{\circ} \mathrm{C} ; b$ flow at $450^{\circ} \mathrm{C}$; $c, d$ are the corresponding experimental flow patterns at $300^{\circ} \mathrm{C}$ and $450^{\circ} \mathrm{C}$ respectively ${ }^{21}$

4 Simulated flow patterns

\section{Results and discussion}

\section{Material flow during extrusion of simple and complex cross-sections}

\section{Rod extrusion}

To evaluate the validity of the FEM simulation to study the effects of material flow at both high and low temperature extrusion, i.e. $450^{\circ} \mathrm{C}$ and $300^{\circ} \mathrm{C}$ respectively, two billets were sectioned along a meridian plane with square grid lines. Similar grid lines were simulated in order to compare with the experimental results from Subramaniyan ${ }^{10}$ and Tutcher. ${ }^{20}$

In the direct extrusion process, a relative movement between the billet and the container exists, encouraging the inhomogeneous nature of the material flow. The effects of the initial billet temperature on material flow for high and low temperature extrusion are illustrated in Fig. $4 a-d$. The figure shows a comparison between the simulated gridded billets at the end of the extrusion (Fig. $4 a$ and $b$ ) and the corresponding macrographs taken from Tutcher's experiments (Fig. $4 c$ and $d$ ). The flow patterns represented by grid lines were originally perpendicular to the billet axis. A good agreement between the experiments and the simulation is obvious. As these figures illustrate, it is clearly evident in both cases that there is a significant change in deformation zone geometry. For high temperature extrusion (i.e. $450^{\circ} \mathrm{C}$ ), the deformation zone gradually spreads from above the die mouth to the container walls by progressive thickening of the zones of heavy shear. The shearing zone occurs in the subcutaneous regions of the billet in preference to sliding at the container wall interface. On the other hand for lower temperature conditions (i.e. $300^{\circ} \mathrm{C}$ ), the extent of this zone is volumetrically less than that observed with higher temperature conditions, the most severe deformation is limited to a small volume close to the die mouth.

Table 2 Rheology data input for computer simulation ${ }^{1}$

\begin{tabular}{llllll}
\hline Alloy & $A$ & $\mathrm{n}$ & $\Delta \boldsymbol{H}$ & $\mathrm{G}$ & $\operatorname{In} \boldsymbol{A}$ \\
\hline AA 2024 & 0.016 & 4.27 & 148880 & 8.314 & 19.6
\end{tabular}



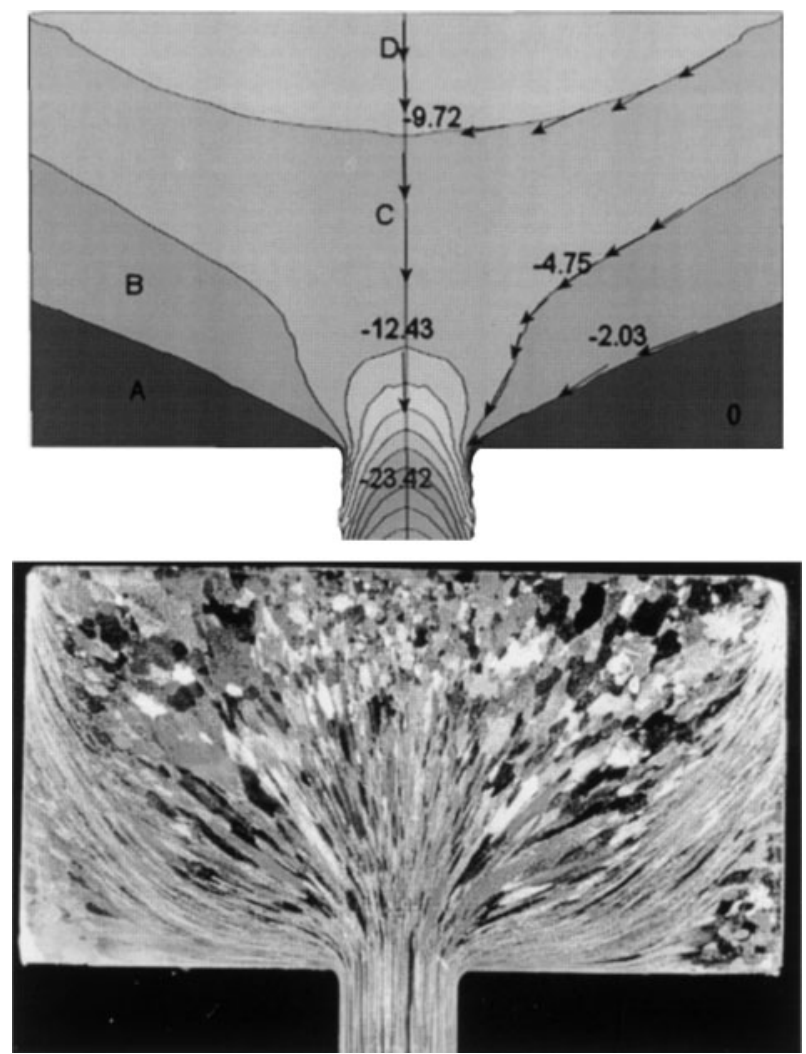

a flow velocity $\left(\mathrm{mm} \mathrm{s}^{-1}\right)$ of material during extrusion at $450^{\circ} \mathrm{C}: A \mathrm{DMZ}, B$ surface generation zone, $C$ main deformation zone, $D$ central deformation zone (initial velocity $\left.3 \mathrm{~mm} \mathrm{~s}^{-1}\right) ; \quad b$ the corresponding experimental micrograph $^{21}$

5 Quasi-static flow of material during extrusion

The formation of the DMZs is evident and over at least $50 \%$ of the volume shown there is no evidence of deformation, the grid is regular across the entire width. Comparing this with the result of the high temperature extrusion shows a considerable difference. In this case, the effects of the deformation can be found over the entire surface shown. Considerable amounts of cylinder wall shear may be seen stretching from the rear of the billet up to the DMZ boundary and progressing further back into the billet, leaving the die mouth flow at a more obtuse angle to the DMZ than the one observed at lower temperatures. The zone of shear produced by friction at a temperature of $450^{\circ} \mathrm{C}$ is greater in depth than the one formed at $300^{\circ} \mathrm{C}$. The shearing at the $\mathrm{DMZ}$ boundary may be regarded as a measure of the work required to provide the force needed to produce a satisfactory surface. This work increases with an enlarged DMZ as a result of the increase of the initial billet temperature and consequent increase in the friction factor at the billet/ container interface. However, it is also the case that surface defects are more easily generated at higher temperatures.

Table 3 Data input and dimensions used for computer simulation

\begin{tabular}{lll}
\hline Initial billet temperature, ${ }^{\circ} \mathbf{C}$ & Shape & Extrusion ratio \\
\hline $300-350$ and 450 & Rod & 30 \\
400 & Square & 40 \\
$350,400-450$ & $T$ & 40 \\
$350-450$ & $U$ & 40 \\
\hline
\end{tabular}
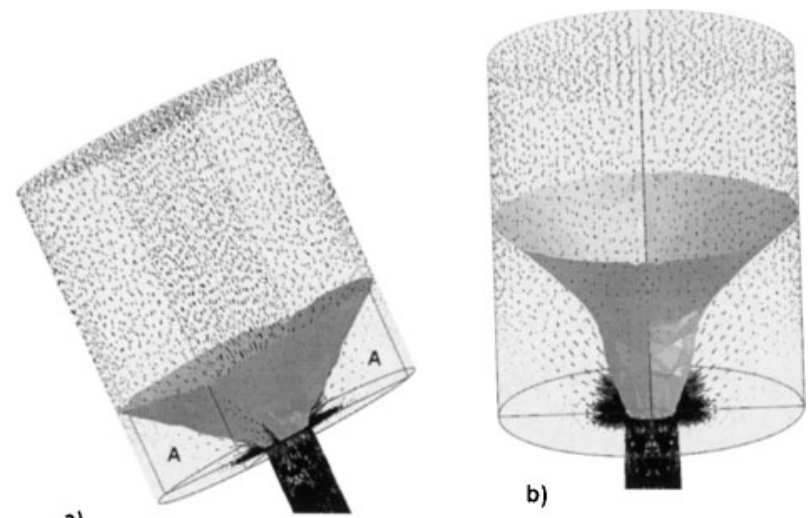

a)

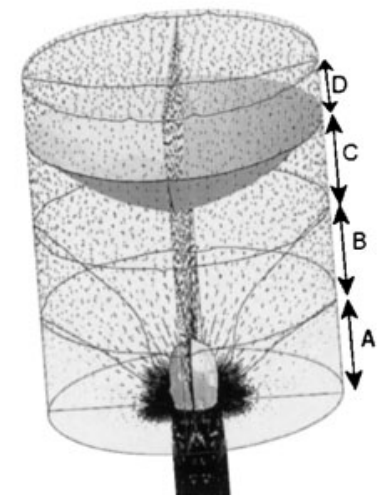

a DMZ; $b$ main deformation zone boundary; $c$ central deformation zone boundary and the corresponding zones outlined in Fig. 5

6 Boundary zones in the quasi-static flow

Therefore, the extent of the DMZ provides some understanding of the influence of temperature on the friction and can be visualised by assessing the velocity profiles. The comparison between the simulation contour lines scalar of material displacement in Forge 3 and the macrograph obtained from Subramaniyan's experiments are shown in Fig. $5 a$ and $b$ respectively. As these figures illustrate, it is clearly evident that there is a good agreement between the experiments and the simulation. As illustrated by Fig. $5 a$, in the regions of the deformation zone close to the die, the central velocity is greater; the rate of travel of material at the billet centre is increasing from the rear of the billet towards the die throat, progressively augmenting the restrained flow of metal near the container wall. The boundaries of the deformation zones illustrated in Fig. 4 shows a smaller volume for the lower temperature extrusion. In addition there is a greater amount of material from the billet subcutaneous layers appearing to reach the extrudate in the high temperature case than the low temperature. This would tend to imply greater diffusion of internal energy dissipation. The temperature gradient between the centre of the billet and the container wall will also cause the shearing layer between the billet and the container to occur at a greater depth below the surface, and so leaves a thicker rim of metal at the billet/container interface. In this connection, the strong adherence of aluminium at lower flow stress to the wall of the container would eventually lead to a very high value of friction.

Therefore, the evidence confirms that a lower temperature extrusion increases the resistance to deformation, reduces the volume of $\mathrm{DMZ}$ and decreases the proportion of the work required to produce the desired surface. 

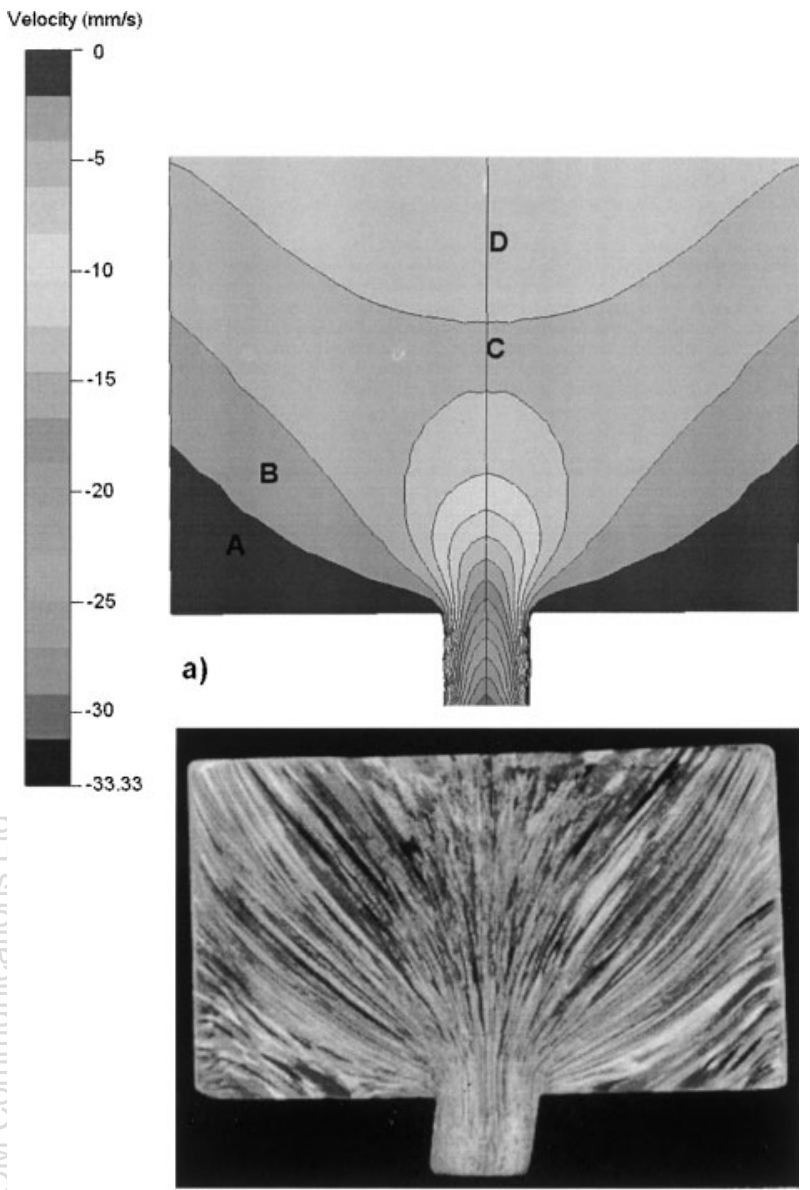

b)

a flow velocity $\left(\mathrm{mm} \mathrm{s}^{-1}\right)$ of material during extrusion at $400^{\circ} \mathrm{C}: A \mathrm{DMZ}, B$ surface generation zone, $C$ main deformation zone, $D$ central deformation zone (initial velocity $\left.3 \mathrm{~mm} \mathrm{~s}^{-1}\right) ; b$ the corresponding experimental micrograph (after Subramaniyan ${ }^{10}$ )

7 Quasi-static flow of material of square section extrusion

The agreement with experimental results validates the simulation model.

\section{Surface generation}

In order to achieve a sound quality surface during the extrusion process, the surface of the extrudate must be generated from within the billet. The generation of this surface involves the production of a virgin surface by shearing of the DMZ. The nature of shear involves production of continuous virgin surfaces at the entrance to the die throat as shown in Fig. 6. However, the boundary conditions at the billet/container interface, i.e. friction, heat transfer, etc., have a significant effect on the material that determines the surface of the extrudate. The quasi-static flow of material during extrusion is shown in Fig. $6 a$. The conditions at the billet interface wall have promoted the formation of a DMZ at the front of the billet. This static volume of material A extends from the die throat, back towards the container wall at an angle, which is dependent on the initial billet temperature and the boundary conditions at the interface. The boundary of this zone A is illustrated in Fig. $6 a$. Outlining this zone is a region of intense shear. The zone resembles the frustrum of a cone in shape and extends from the die throat to the container wall as

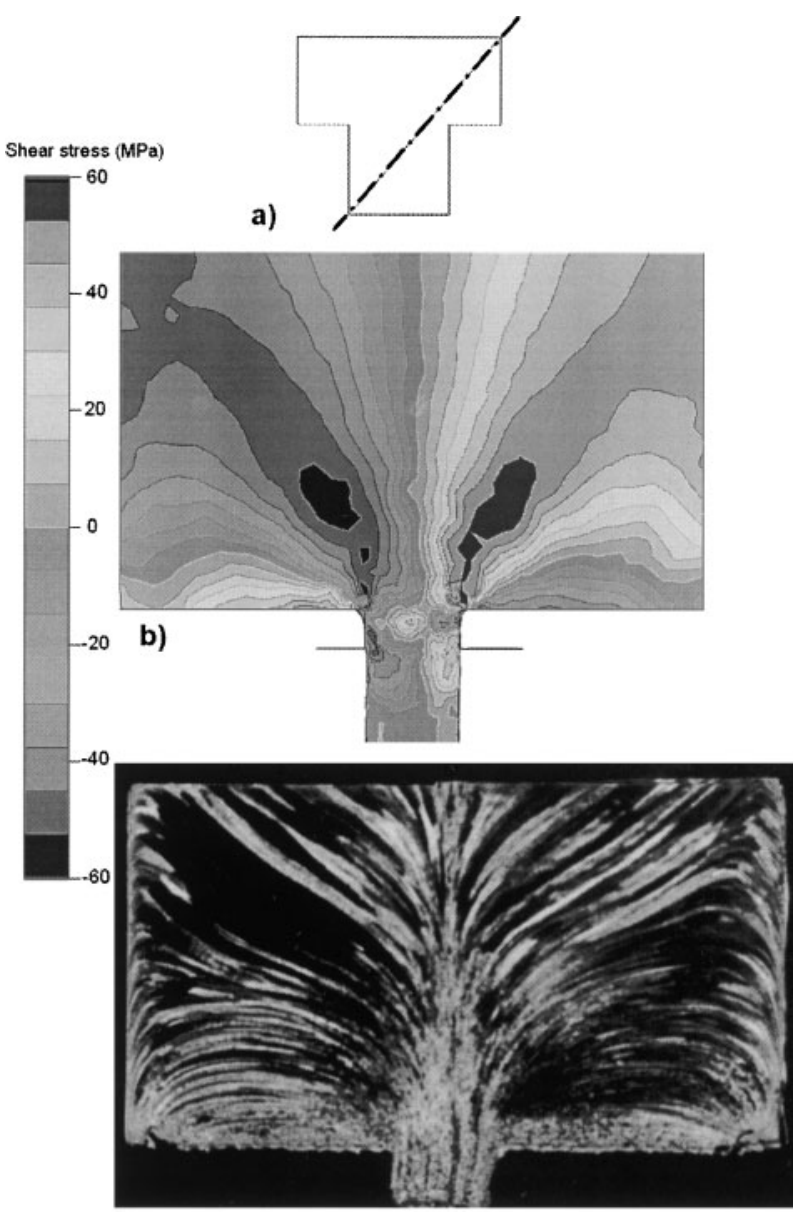

c)

$a$ the plane of $T$ die from which the billet was sectioned used in the experiments and the simulation; $b$ shear stress; $c$ the corresponding experimental micrograph (after Subramaniyan ${ }^{10}$ ) (initial velocity $3 \mathrm{~mm} \mathrm{~s}^{-1}$ )

$8 \mathrm{~T}$ section extrusion showing the asymmetry of material during quasi-static flow

illustrated in Fig. $6 b$. It can be seen from Fig. $6 a$ that the extrudate material originates from various locations in the deforming billet, which may be represented by three zones. The surface generation zone $\mathrm{B}$ outlining the $\mathrm{DMZ}$, the main deformation zone $\mathrm{C}$ and the central deformation zone $\mathrm{D}$. The outer surface of the extrudate originates from the surface generation zone $B$ as illustrated by Fig. $6 a$. It is material from this zone moving along the DMZ boundary that forms the extrudate surface. The figure also suggests that the outside surface of the extrudate originates from the material, which is located on the edge of the DMZ and moves more slowly into the extrudate surface promoting a continuous formation of a virgin surface. The material forming the core of the extrudate originates from the central deformation zone D of heavy shear feeding into the bulk of the extrudate as illustrated also in Fig. $6 c$. This central deformation zone supplements the material feeding the body of the extrudate from within the main deformation zone $\mathrm{C}$.

\section{Section shapes}

The effect on the material flow of changing the section geometry from circular to square is shown in Fig. $7 a$ and $b$. The figure shows a comparison between the predicted 

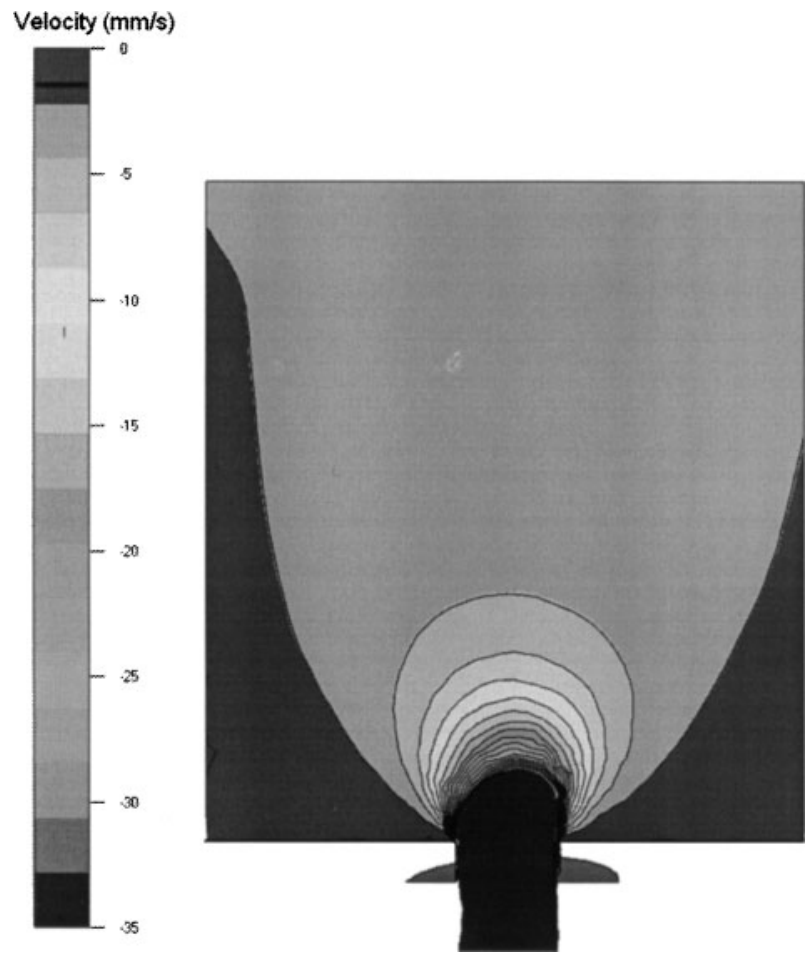

9 Asymmetry in material flow in the $T$ section represented by the velocity profile (initial velocity $v=3 \mathrm{~mm} \mathrm{~s}^{-1}$ )

contour lines scalar of the shear stress using Forge 3 (Fig. 7a) and the macrographs of a partially extruded billet of super purity aluminium obtained from experiments ${ }^{10}$ (Fig. $7 b$ ). The planar section shown was taken along the diagonal of the square. As these figures illustrate, it is clearly evident that there is a good agreement between the experiments and the simulation. Similar to the material flow in a circular cross-section, the flow patterns resemble a typical direct extrusion flow with the fast moving central zone outlined by a slow moving restrained periphery as shown in Fig. $7 a$. Both the simulation and experimental macrographs of Figs. 5 and 7 exhibited no significant differences in the material

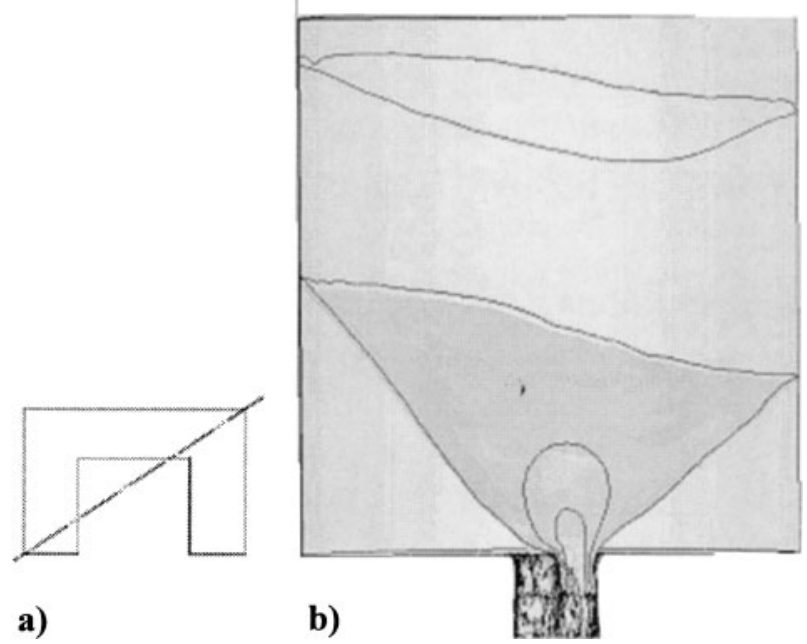

10 (a) The plane of $U$ die from which the billet was sectioned in the experiments and the simulation; (b) $U$ section extrusion showing the asymmetry flow during quasi-static deformation

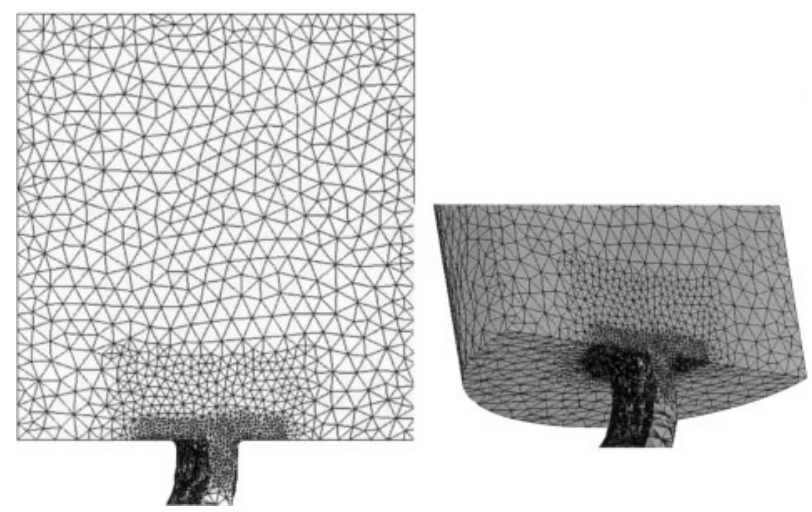

11 The material does not flow in a radial plane along the longitudinal axis of the die as a result of a non-uniform stress and strain distribution in the deforming material

flow between the two sections. This is expected because the material flow in a square section is analogous to the flow in circular sections, mainly because there is no severe change in the symmetry of the section between a square and a circle. The origin of the extrudate surfaces remains the same as those observed by rod extrusion.

The experimental and simulation of the quasi-static deformation flow corresponding to the extrusion of $\mathrm{T}$ sections are shown in Fig. $8 b$ and $c$ respectively. The simulation patterns, in Fig. 8, are presented by the shear stress distribution on the sectioning plane. The plane from which the billet was sectioned is shown in Fig. $8 a$. It can be seen that the flow patterns are slightly different from the experimental patterns in contrast to those obtained for rod and square sections. This small difference between the experimental and the simulation flow patterns could be attributed to the material, super pure aluminium (experiments) and AA 2024 (simulations) used to investigate material flow visualisation. The super pure aluminium is particularly suited to study deformation behaviour because of its large grain size and ease of macroetching. This probably demonstrates that a significant difference in the flow characteristics can exist with alloy composition especially for complex sections. Another possible reason for this difference could be explained from the sectioning method used for flow visualisation for both experimental and simulation. The downside of the sectioning method is that a slight deviation from the required line in cutting the section will result in a different area of the flow pattern being visualised. This is one of the disadvantages of the sectioning method used in experiments to investigate the material flow. The changes in the deformation patterns indicate that, to some degree, the variations in profile across the section may contribute to changes in the flow patterns. It is evident from the experimental illustrations that there are some differences in representing the material flow for complex shapes.

The material flow in the $\mathrm{T}$ shape exhibited similar characteristics to that of the rod and square extrusion. It can be seen clearly in Fig. 8 that the region of the intense shear feeding the extrudate surface extends from the die face, adjacent to the die mouth, to the container/billet interface located towards the rear of the billet. However, a certain amount of asymmetry about the billet axis can be seen, especially near the DMZ as illustrated by the 


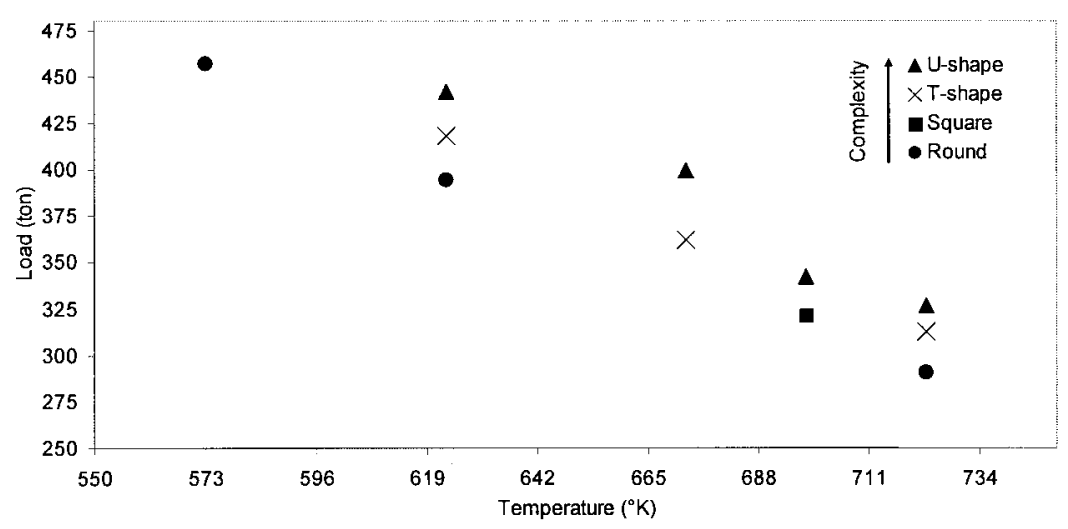

12 The effect of section geometry on the maximum extrusion load at different extrusion temperatures

velocity profile in Fig. 9. Similar behaviour was observed in the extrusion of the $U$ section as shown in Fig. 10, demonstrating that in terms of complexity of flow both sections $\mathrm{T}$ and $\mathrm{U}$ are the same. This irregularity about the billet axis is due to the inhomogeneous nature of the material flow encountered when extruding complex sections. The distance over which the material flows on the transverse and the longitudinal axis on both sides of the section geometry is different causing asymmetry of the flow patterns. The material flow does not remain in a radial plane along the longitudinal axis of the die and this behaviour results in a non-uniform stress and strain distribution in the deforming material resulting in a slightly curved extrudate (Fig. 11), which could be improved by correcting the die land length around the perimeter.

\section{Effect of section shape on peak pressure}

The effect of the die geometry on the computed maximum extrusion load is shown in Fig. 12. The figure illustrates that for the same extrusion ratio of 40:1 and extrusion speed of $3 \mathrm{~mm} \mathrm{~s}^{-1}$ and over a range of temperatures considered, the peak extrusion load increment is increased with increasing complexity in the extruded section. It should be noted that the pressuredisplacement curves have not been represented in this paper because the trends observed are similar to that of round cross-section. ${ }^{3,4,10-12,22}$

This increase in load with the increase in section complexity can be attributed to several interrelated reasons in which the complexity of extrusion increases the resistance to deformation. The increase in load is

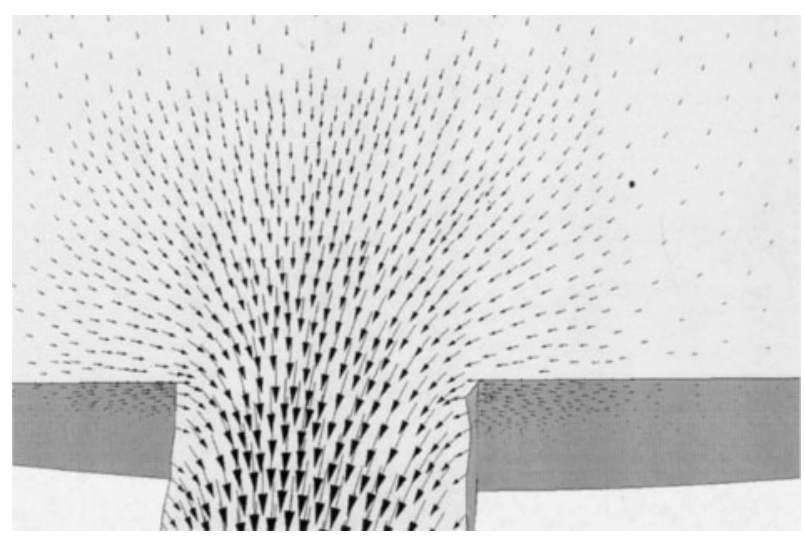

13 T section. Different views illustrating the bending of the material flow lines at the die entrance required to overcome the increase in frictional resistance and complexity flow associated with the increase in the contact area in the die land regions. The deformation zone becomes more intricate and larger in shape as the section geometry diverges from simple rounds to complex shapes. The material approaching the die corners is divided, forming regions of divergent flow and as a consequence, promoting localised distortions at the die entry as illustrated in Fig. 13 (for example ' $T$ ' section). This material flow is associated with the bending of the flow lines into the die as a result of reentrant corners similar to the events occurring at the inlet and outlet regions of the deformation zone. The extrudate surfaces also suffer a higher degree of deformation with the increase in section complexity, and as a consequence a further load is required to overcome the region of intense shear feeding the extrudate surfaces.

Irrespective of the section geometry, as shown in Fig. 14, the material forming the extrudate originates

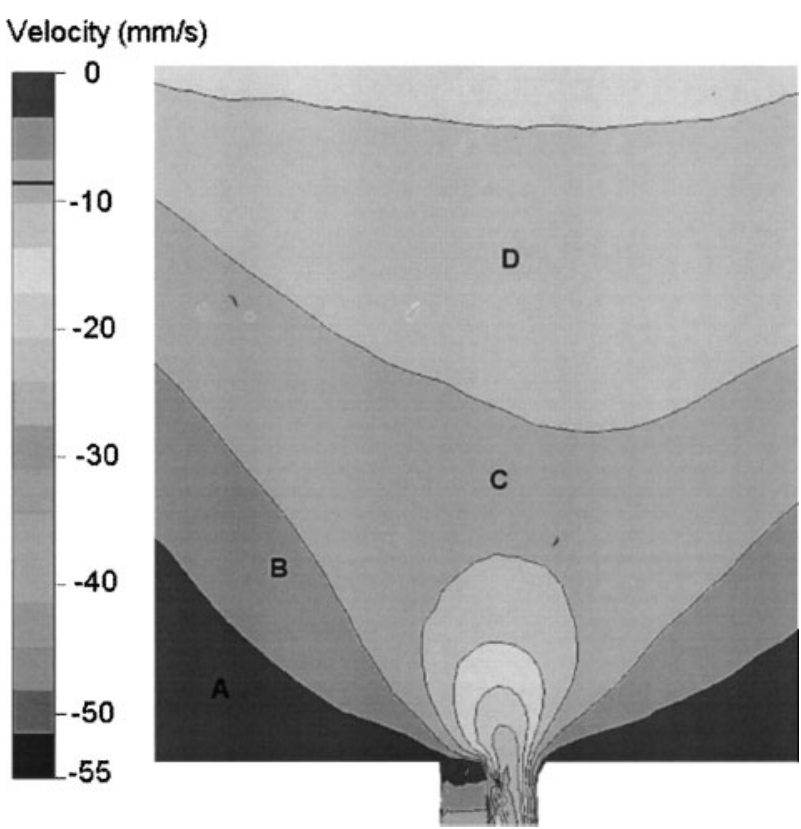

$A \mathrm{DMZ}$; $B$ surface generation zone; $C$ main deformation zone; $D$ central deformation zone (initial velocity $v=3 \mathrm{~mm} \mathrm{~s}^{-1}, R=40: 1$ and initial billet temperature is $\left.300^{\circ} \mathrm{C}\right)$

14 Boundary zones in the quasi-static flow of the $U$ section extrusion represented by the velocity profile 
from various locations in the deforming billet similar to those observed during the rod extrusion.

\section{Conclusions}

1. FEM simulation successfully predicted the origin of differing sections of the extrudate that flows from varying portions of the deformation zones.

2. In the current investigation it has been shown that the prediction of material flow and the DMZ formation were mostly in good agreement between simulation and experimental finding.

3. The finite element programs Forge 2 and Forge 3 appear to predict all the major characteristics of the flow observed macroscopically.

4. Further work is required to model the die land volume such that the extrudate emerges straight.

\section{Acknowledgement}

The authors would like to express their thanks to Dr J. Subramaniyan and M. G. Tutcher and recognise the excellent contribution from their experimental work. The provision of facilities by Bournemouth University is also gratefully recognised.

\section{References}

1. T. Sheppard: 'Extrusion of aluminium alloys'; 1999, Dordrecht, Kluwer.

2. E. P. Wood and T. Sheppard: Aluminium, 1975, 51, pp.760-764.

3. I. Flitta and T. Sheppard: Mater. Sci. Technol, 2002, 18, 987-994.

4. I. Flitta and T. Sheppard: Mater. Sci. Technol., 2003, 19, 837-846.

5. H. Valberg and T. Malvik: Int. J. Mater. Prod. Technol., 1994, 9, 428-463.
6. H. Valberg: J. Mater. Proc. Technol., 1993, 31, 39-55.

7. M. P. Clode and T. Sheppard: The Aluminium Technology, 230240; 1986, London, Institute of Metals.

8. T. Sheppard and E. P. Wood: J. Metals Technol., 1980, 7(2), 58-66.

9. G. Grasmo, K. Holte, S. Støren, H. Valberg, R. Flatval, L. Hanssen, M. Lefstad, O. Lohne, T. Web, R. Ørsund and J. Herberg: Proc. 5th Int. Seminar on 'Aluminum extrusion technology', Chicago, 367-376; 1992, Washington, DC, The Aluminum Association.

10. J. Subramaniyan: 'Extrusion of 2024 aluminium alloy sections', $\mathrm{PhD}$ thesis, Imperial College, London, 1989.

11. I. Flitta and T. Sheppard: Proc. 7th Int. Seminar on 'Aluminum extrusion technology', Chicago, Vol. 1, 197-203; 2000, Washington, DC, The Aluminum Association.

12. I. Flitta and T. Sheppard: Proc. 5th Int. ESAFORM Conf. on 'Materials forming', Krakow, Poland, 2002, 435-438.

13. R. J. Dashwood, H. R. McShane and A. Jackson: Proc. 6th Int. Seminar on 'Aluminum extrusion technology', Chicago, 331-339; 1996, Washington, DC, The Aluminum Association.

14. M. Laroussi and L. Fourment: Proc. 5th Int. EESAFORM Conf. on 'Materials forming', Krakow, Poland, 2002, 19-21.

15. A. J. Williams, T. N. Croft and M. Cross: Proc. 5th Int. EESAFORM Conf. on 'Materials forming', Krakow, Poland, April 2002, 67-70.

16. J. L. Chenot and M. Bellet: in 'Numerical modelling of material deformation processes, research, development and applications', (eds. P. Hartley, I. Pilling and C. Sturgess), 179-224; 1992, London, Springer.

17. C. M. Sellars and W. J. McG. Tegart: Int. Metall. Rev., 1972, 17, 124.

18. T. Sheppard and D. Wright: J. Metal Technol., 1979, 6, 215-223.

19. T. Sheppard and A. Jackson: Mater. Sci. Technol., 1997, 13, 203209.

20. M. G. Tutcher: 'Deformation processing applied to the $\mathrm{Al}-\mathrm{Mg}$ alloy system', PhD thesis, Imperial College, London, 1979.

21. T. Sheppard and M. G. Tutcher: Metals Sci., 1980, 14, 579-589.

22. T. Flitta and T. Sheppard: Proc. 8th Int. Seminar on 'Aluminum extrusion technology', May 2004, 269-283; Washington, DC, The Aluminum Association. 